\title{
DYNAMIC COMPRESSION OF POLYETHYLENE FOAM WITH TRAPEZOIDAL CROSS-SECTION
}

\author{
P. Osowski* ${ }^{*}$ M. Wolski ${ }^{* *}$, T. Piątkowski ${ }^{* * * *}$
}

\begin{abstract}
In packaging the cushioning materials are used, for product protection from shock. The most commonly used protections are rib structures with trapezoid cross-section made from PE foam. The paper concerns about analysis of rib shape influence on the dropping platen maximal acceleration, during impact with cushioning material. The research was conducted for various side wall inclination angle of ribbed sample, foam thickness and free fall height of dropping platen. The research results show that using sample with trapezoidal cross-section, the higher acceleration is obtained than in the case of cuboidal samples of the same volume.
\end{abstract}

Keywords: trapezoidal cross-section, cushioning curves, impact, foam, packaging

\section{Introduction}

The use of cushioning materials is a necessity no matter if has to protect a product against consequences of collision during transport, a passenger in a car, or the head of the cyclist. Polymer foams belongs to the cushioning materials that are the most commonly used in packaging due to fact that they are cheap in production and effectively absorb impact energy. In production process, they can be easily shaped and adapted to the complex geometry of the product.

Classic method of packaging design using foams is based on using cushioning curves (Fiedler, 2009). These curves are determined on the basis of experiment research consisting in measuring maximal acceleration of dropping platen, when it collides with the cuboidal foam sample. From the analysis of cushioning curves, we know what thickness and supporting surface should cushioning material have, so that maximum acceleration experienced by the product during the free fall from desired height does not exceed a predetermined value. When combining this method with packaging drop test, the impact factor (the ratio between the maximum acceleration to the gravitational acceleration) can be predicted for packaging subjected to impact. However, this method cannot be directly used in ribbed packaging structures design.

In the BASF company information materials (BASF, 2006) is specified that to acquire the same maximum acceleration in a ribbed sample with a trapezoidal cross-section during impact as in the cuboidal sample with surface $A$, the side wall inclination angle should be $15^{\circ}$ and the surface in the half of the rib thickness should be A. Numerical analysis was carried out in paper (Masso-Moreu Y. et al., 2003) that proves the validity of these construction assumptions, however, they did not answer the question of how the acceleration changes when the angle increase.

The purpose of this article is analysis influence of the inclination angle of the trapezoidal cross-section side wall on the obtained maximum acceleration in the case of ribbed cushioning structure.

M. Eng. Przemysław Osowski: Faculty of Mechanical Engineering, UTP University of Science and Technology, Al. Prof. S. Kaliskiego 7, 85-796, Bydgoszcz; Poland, przoso000@utp.edu.pl

** M. Eng. Mirosław Wolski: Faculty of Mechanical Engineering, UTP University of Science and Technology, Al. Prof. S. Kaliskiego 7, 85-796, Bydgoszcz; Poland, Miroslaw.Wolski@utp.edu.pl

*** Assoc. Prof. Tomasz Piątkowski, PhD.: Faculty of Mechanical Engineering, UTP University of Science and Technology, Al. Prof. S. Kaliskiego 7, 85-796, Bydgoszcz; Poland, topiat@utp.edu.pl 


\section{Object of research}

The object of research is PE foam having an isosceles trapezoidal cross-section (Fig. 1a). The length of the sample is $L=100 \mathrm{~mm}$, while the thickness is $t=[20,40] \mathrm{mm}$. Side walls inclination angle of the sample relative to $z$ axis was used $\alpha=[0,15,30,45,60]^{\circ}$. The foam base dimensions $b$ and $c$ are determined using assumption, that at the half of the thickness $t$ the dimension $d$ has a fixed value $(d=100$ $\mathrm{mm})$. Using the angle $\alpha$ we can determine dimension $b$ by the equation:

$$
b=d-t(\operatorname{tg} \alpha)
$$

and the dimension $c$ with equation:

$$
c=d+t(\operatorname{tg} \alpha)
$$

The fixed dimension $d$ causes that the samples have fixed volume and only the angle influence on the obtained maximum acceleration is researched.

a)

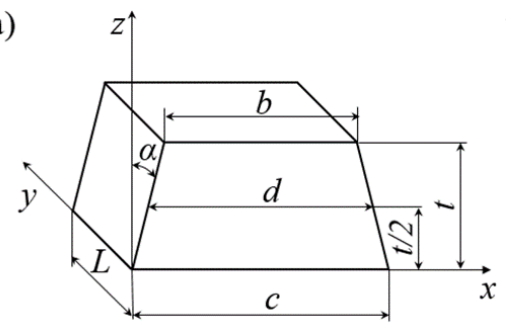

b)

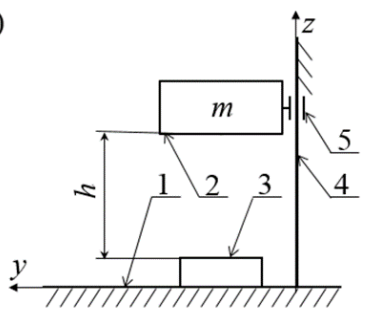

Fig. 1: a) PE foam with trapezoidal cross-section with basic dimensions, b) impact tester; 1 - rigid surface, 2 -dropping platen with acceleration recorder, 3 - sample, 4 - guideway, 5 - linear bearing.

To determine the maximum acceleration, the impact tester (Fig. 1b) was used, to test the dynamic properties of cushioning materials. This tester was presented in paper (Osowski et al., 2017). This device consists of a dropping platen with acceleration recorder (2), which free fall from specified height $h$ on tested sample (3), placed on the rigid surface (1).

\section{Research results}

The maximum values of accelerations recorded by the impact tester dropping platen are presented as a function of the side wall inclination angle $\alpha$ (Fig. 2). Accelerations were determined at the given sample thickness $t$ and the free fall height $h$ of dropping platen.
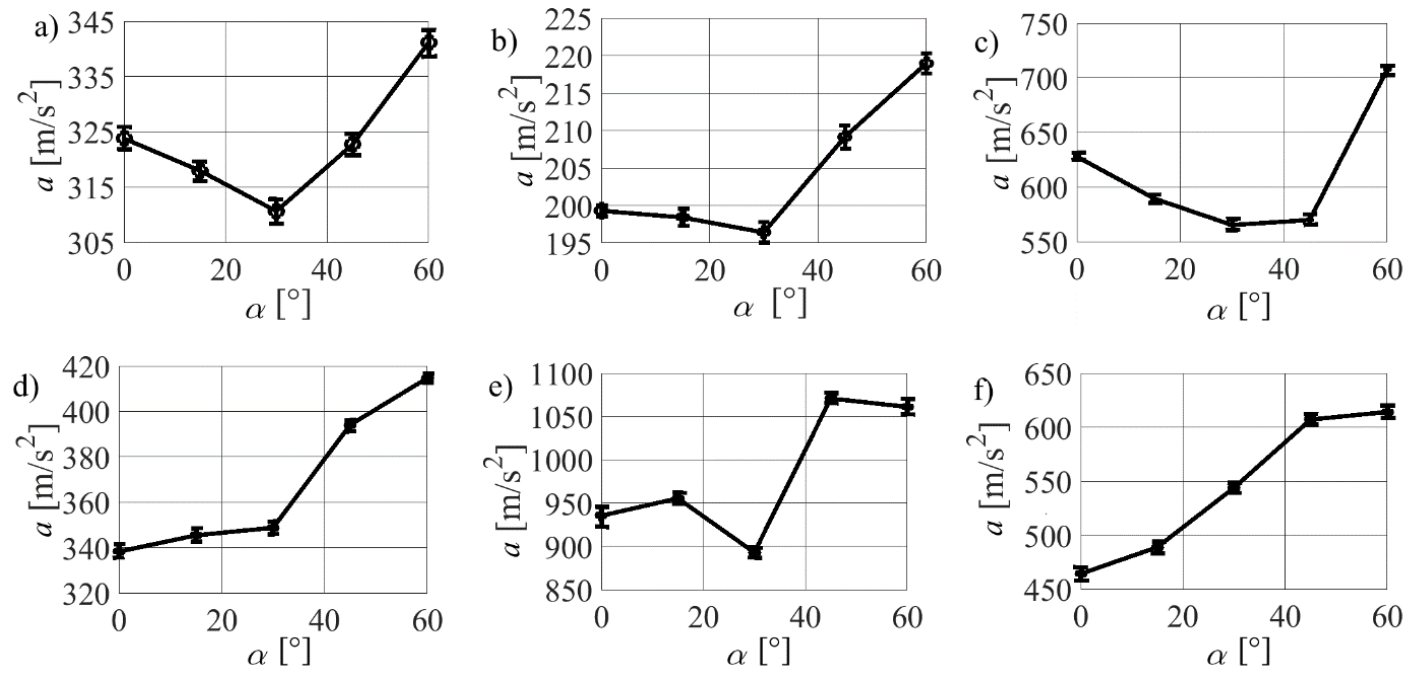

Fig. 2: Chart of maximum acceleration as a function of side wall inclination angle, when:

a) $h=0.15 \mathrm{~m}, t=20 \mathrm{~mm}, b) h=0.15 \mathrm{~m}, t=40 \mathrm{~mm}, c) h=0.30 \mathrm{~m}, t=20 \mathrm{~mm}, d) h=0.30 \mathrm{~m}$, $t=40 \mathrm{~mm}, e) h=0.45 \mathrm{~m}, t=20 \mathrm{~mm}, \mathrm{f}) \mathrm{h}=0.45 \mathrm{~m}, t=40 \mathrm{~mm}$. 
For each measurement point the confidence interval was determined, using the averaged values of acceleration (determined from 16 trials) at a confidence level of 95\%. As can been seen, for samples with thickness $t=20 \mathrm{~mm}$ (Fig. 2a, c, e) there is a decrease in the acceleration in the angle range to $30^{\circ}$, and after exceeding this value, there is a rapid increase. For thickness $t=40 \mathrm{~mm}$ (Fig. 2b, d, f) there is the same dependency only for the free fall height $h=0.15 \mathrm{~m}$.

Using data from charts, a Fig. 3a was draw up. This chart represents acceleration $a$ as a function of angle $\alpha$ and energy density $E_{d}$ delivered to the structure - specifying angle $\alpha$, thickness $t$ and height $h$ influence on the achieved maximum acceleration. Energy density is defined as the ratio of the potential energy of the dropping platen to the volume of the tested sample (Li G. et al., 2015):

$$
E_{d}=\frac{m g h}{L d t}
$$

where:

$m$ - dropping platen mass $(m=4.405 \mathrm{~kg})$,

$g$ - acceleration of gravity,

$h$ - free fall height $(h=[0.15,0.30,0.45] \mathrm{m})$,

$L, d, t$ - dimensions shown in Fig. 1a $(L=100 \mathrm{~mm}, d=100 \mathrm{~mm}$ and $t=[20,40] \mathrm{mm})$.

Table 1 is presenting juxtaposition of energy densities obtained during tests for individual thicknesses and free fall heights.

Tab. 1: Juxtaposition of energy density $E_{d}\left[\mathrm{~J} / \mathrm{mm}^{3}\right]$ obtained during tests depending on the free fall height $h$ and sample thickness $t$

\begin{tabular}{cccc}
\hline \multirow{2}{*}{$\boldsymbol{t}[\mathrm{mm}]$} & 0.15 & \multicolumn{3}{c}{$\boldsymbol{h}[\mathrm{m}]$} \\
\cline { 2 - 4 } & $3.24 \times 10^{-5}$ & 6.30 & 0.45 \\
\hline 20 & $1.62 \times 10^{-5}$ & $3.24 \times 10^{-5}$ & $9.72 \times 10^{-5}$ \\
\hline 40 & &
\end{tabular}

From analysis of Fig. 3a, it can be seen that with increasing angle $\alpha$ the achieved maximum acceleration $a$ also increases. In addition, the magnitude of this change depends on the obtained energy density.

a)

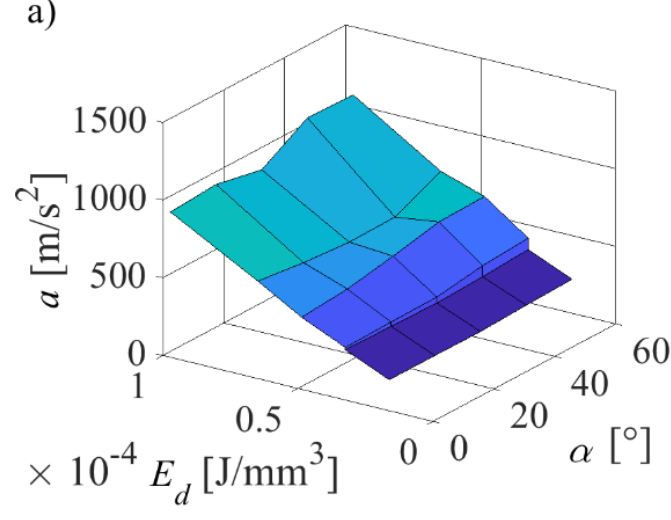

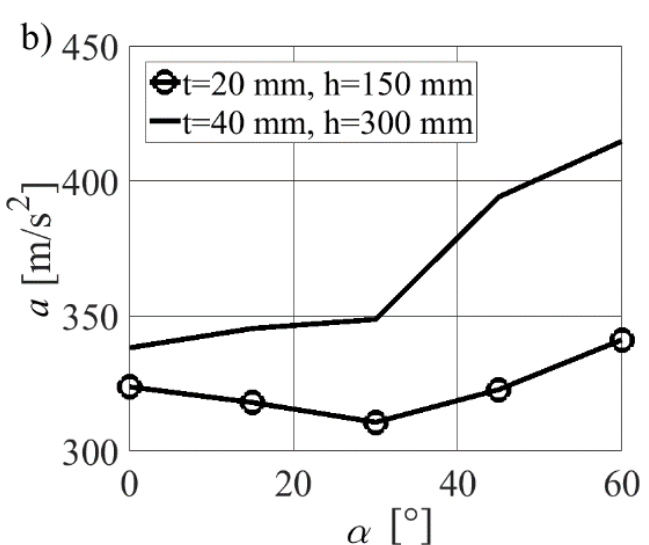

Fig. 3: a) Chart of maximum acceleration as a function of side wall inclination angle and energy density, b) chart of maximum acceleration as a function of side wall inclination angle for an energy density equal $E_{d}=3.24 \times 10^{-5}\left[\mathrm{~J} / \mathrm{mm}^{3}\right]$ 
In Fig. 3a for energy density equal $E_{d}=3.24 \times 10^{-5}\left[\mathrm{~J} / \mathrm{mm}^{3}\right]$, accelerations for two cases are presented: $t=20 \mathrm{~mm}$ and $h=0.15 \mathrm{~m}$ also $t=40 \mathrm{~mm}$ and $h=0.30 \mathrm{~m}$ (acc. tab. 1). As can been seen, at the same energy density (Fig. 3b), maximum accelerations between each other are different - causing a step change in the value shown in Fig. 3a. In addition, at $t=40 \mathrm{~mm}$ there is an increase of acceleration up to angle $\alpha=30^{\circ}$, and at $t=20 \mathrm{~mm}$ is going down.

\section{Conclusions}

Maximum acceleration $a$ for foam with trapezoid cross-section has been determined as a function of side wall inclination angle $\alpha$, free fall height $h$ and material thickness $t$. It turned out that with the increase of the angle $\alpha$ to $30^{\circ}$, acceleration $a$ may show a slight decreasing trend (e.g. Fig $3 \mathrm{~b}$, for $t=20 \mathrm{~mm}$ ) or growing (e.g. Fig 3b, for $t=40 \mathrm{~mm}$ ). However, in the angle range $\alpha \in\left\langle 30^{\circ} ; 60^{\circ}\right\rangle$ acceleration $a$ increases for both $t=20 \mathrm{~mm}$ and $t=40 \mathrm{~mm}$. This is due to the complex state of strains appearing in the upper part of the sample (near dimension $b$ ) (Gilchrist A. et al., 2001). When using large angle $\alpha$ values, the dimension $b$ is getting smaller than the dimension $c$ of the trapezoidal cross-section. The upper part of the sample (close to dimension $b$ ) is much more deformed than the lower part (close to dimension $c$ ). Given the progressive characteristic of dynamic stress-strain curve of the PE foam material that was presented in paper (Osowski et al., 2017), it is expected that with a significant increase of strain, very high stresses appear in the upper part of the sample. This state finally causes getting higher acceleration $a$, than a cuboid sample with the same volume and obtaining different accelerations while maintaining a constant energy density value and weight of the dropping platen.

On the basis of the conducted research, it can be stated, that the use of very large inclination angles of the rib walls leads to unfavorable stress distribution in the ribs, contributing to the reduction of the cushioning properties of the packaging.

\section{References}

Basf brochure Packaging with Styropor. Ludwigshafen, Germany, 2006. http//styropor.basf.us/files/pdf/ Packaging_with_Styropor.pdf (date of download 16 January 2018).

Fiedler R. M. (2009) Shock, In: Yam K.L., Encyclopedia of packaging technology. John Wiley \& Sons, pp. 11071111 .

Gilchrist. A., Mills N.J. (2001), Impact deformation of rigid polymeric foams: experiments and FEA modelling, International Journal of Impact Engineering, 25, pp. 767-786

Li G., Rouillard V. and Sek M. A. (2015) Evaluation of Static and Dynamic Cushioning Properties of Polyethylene Foam for Determining Its Cushion Curves, Packaging Technology and Science, 28, pp. 47-57.

Masso-Moreu Y. and Mills N.J. (2003) Impact compression of polystyrene foam pyramids, International Journal of Impact Engineering, 23, 6, pp. 653-676.

Osowski P. and Piątkowski T. (2017) Analysis of corrugated cardboard influence on the protective properties of complex packaging system, AIP, In: Conference Scientific Session of Applied Mechanics IX (ed. Tomasz Piątkowski), UTP, Bydgoszcz pp. 020011-1 - 020011-9. 